\title{
Europa in der Corona-Krise: Europäische Lieferketten müssen europäisch wiederbelebt werden
}

In allen europäischen Staaten werden aufgrund der CoronaPandemie und der entsprechenden Gegenmaßnahmen historische Einbrüche der Wirtschaftsleistung erwartet. Dem Consensus Forecast Mai 2020 zufolge könnte das diesjährige Bruttoinlandsprodukt in Deutschland um 6,3\%, in

(C) Der/die Autor(en) 2020. Open Access: Dieser Artikel wird unter der Creative Commons Namensnennung 4.0 International Lizenz (https:// creativecommons.org/licenses/by/4.0/deed.de) veröffentlicht.

Open Access wird durch die ZBW - Leibniz-Informationszentrum Wirtschaft gefördert.

* Die Autoren danken Esther Thiel für die tatkräftige Unterstützung bei der Erstellung der Abbildungen.
Frankreich um 8,2\%, in Italien um 9,9\% und in Spanien um 9,1\% zurückgehen. Die EU-Mitgliedstaaten sind dabei auf unterschiedliche Weise betroffen und befinden sich in unterschiedlichen Ausgangssituationen. Über mögliche europäische Maßnahmen wird heftig gerungen. Dabei sollte immer im Blick bleiben, dass Europa seine Wirtschaft aufgrund seiner engen Verflechtung durch den Binnenmarkt nur gemeinsam wiederbeleben kann. Dafür bedarf es geeigneter Rahmenbedingungen und Maßnahmen auf europäischer Ebene.

Etwa $17 \%$ der deutschen Wertschöpfung findet über globale Wertschöpfungsketten statt (Flach et al., 2020). Vor allem das Verarbeitende Gewerbe ist dabei über den Bezug von Vorleis- 
tungen und den Export von Gütern global verflochten, insbesondere mit anderen EU-Mitgliedstaaten. Entsprechend bedeutend ist der freie Warenverkehr für Deutschland. Um internationale Wertschöpfungsketten schnellstmöglich wiederzubeleben, bedarf es einer effektiven Koordination internationaler, europäischer und nationaler Maßnahmen. Als Leitlinie könnte hier das europäische Subsidiaritätsprinzip dienen.

\section{Wirkungskanäle der globalen Pandemie}

Durch die weltweite Ausbreitung der Pandemie sind mit Europa, Nordamerika und Asien diejenigen Regionen betroffen, die am stärksten über internationale Wertschöpfungsketten miteinander verflochten sind. Die Pandemie sowie die zu ihrer Eindämmung beschlossenen gesundheitspolitischen Maßnahmen sind weltweit mit höchster Unsicherheit für die Unternehmen verbunden, sowohl bezüglich des Gesundheitsrisikos als auch bezüglich der Geschäftserwartungen. Die Geschäftstätigkeit vieler Unternehmen ist durch die Einführung umfangreicher Beschränkungen des öffentlichen Lebens sowie durch Vorsichtsmaßnahmen für Mitarbeiterinnen und Mitarbeiter sowie Kundinnen und Kunden zum Teil signifikant eingeschränkt.

Die Auswirkungen der pandemiebedingten Einschränkungen auf die Wertschöpfungsketten lassen sich über vier Kanäle charakterisieren, die für nationale sowie internationale Zuliefer- und Endkundenbeziehungen relevant sind:

- Ausbleibende Vorleistungszulieferungen etwa aufgrund von Werksschließungen in vorgelagerten Wertschöpfungsstufen sowie Hemmnissen im internationalen Warenverkehr: Diese Sorge trieb viele Unternehmen vor allem zu Anfang der Pandemie um, als mit China ein wichtiges Ursprungsland für Vorleistungen betroffen war.

- Ausbleibende Nachfrage nach Vorleistungen aufgrund von Werksschließungen in nachgelagerten Wertschöpfungsstufen: So führten Werksschließungen der großen Automobilhersteller zu Nachfrageausfällen bei vorgelagerten Zulieferunternehmen, die daraufhin ihre Produktion drosselten.

- Ausbleibende Nachfrage nach Investitionsgütern: Bei vielen Unternehmen dürfte die Pandemie zu einer Reduktion der zu erwartenden Nachfrage und somit zu geringeren geplanten Investitionen geführt haben. Weiterhin dürfte die gestiegene Unsicherheit über die zukünftige Entwicklung zu einem „wait-and-see“-Verhalten, also einer Verschiebung von Investitionsprojekten führen (Bachmann und Bayer, 2013).

- Ausbleibende Konsumnachfrage: Einerseits führten staatlich angeordnete Schließungen im Handel und Dienstleis-
Dr. Jan Fries, Niklas Garnadt, M.Sc., und Lukas Nöh, Ph.D., sind Mitglieder des wissenschaftlichen Stabs beim Sachverständigenrat zur Begutachtung der gesamtwirtschaftlichen Entwicklung (SVR).

Prof. Dr. Veronika Grimm ist Professorin an der Friedrich-Alexander-Universität Erlangen-Nürnberg und Mitglied im SVR.

tungsgewerbe zu Rückgängen der Konsumnachfrage. Andererseits dürfte ein Rückgang der verfügbaren Einkommen und die Unsicherheit über die zukünftigen Einkommen zu Konsumzurückhaltung führen.

\section{Die hohe Vorleistungsverflechtung im Verarbeitenden Gewerbe}

Das Problem gestörter Lieferketten und infolgedessen ausbleibender Vorleistungszulieferungen wiegt insbesondere im Verarbeitenden Gewerbe schwer. Während in den Bereichen Handel und Dienstleistungen weniger als $40 \%$ des Produktionswerts auf Vorleistungen zurückgeht, beträgt dieser Anteil im Verarbeitenden Gewerbe $65 \%$ (vgl. Abbildung 1). Die internationale Verflechtung in den Bereichen Handel und Dienstleistung ist mit einem Anteil der importierten Vorleistungen am Produktionswert von lediglich $4 \%$ erheblich geringer als im Verarbeitenden Gewerbe mit $24 \%$, was etwa einem Drittel der gesamten Vorleistungen entspricht.

Etwa zwei Drittel der importierten Vorleistungen im Verarbeitenden Gewerbe stammen dabei aus einem Mitgliedstaat der EU. Eine ähnliche Verflechtungsstruktur des Verarbeitenden Gewerbes ist in den meisten anderen EU-Mitgliedstaaten zu beobachten. In 20 der 27 Mitgliedstaaten werden mehr als die Hälfte der importierten Vorleistungen aus anderen Mitgliedstaaten importiert. Dies verdeutlicht die starke Verflechtung innerhalb der EU sowie die hohe Bedeutung, die funktionsfähige Lieferketten für die Wertschöpfung im Verarbeitenden Gewerbe besitzen.

Die Bedeutung importierter Vorleistungen weist dabei eine deutliche Heterogenität zwischen den einzelnen Wirtschaftszweigen des Verarbeitenden Gewerbes auf. Die Spannweite des Anteils importierter Vorleistungen an den gesamten Vorleistungen in den bruttowertschöpfungsstärksten Wirtschaftszweigen reicht von etwa $20 \%$ in der Nahrungsmittelverarbeitung bis etwa $45 \%$ in der chemischen Industrie (eigene Berechnungen auf Basis der World Input Output Database; Timmer et al., 2015). In all diesen Wirtschaftszweigen 
Abbildung 1

Vorleistungsanteile der deutschen Wirtschaft

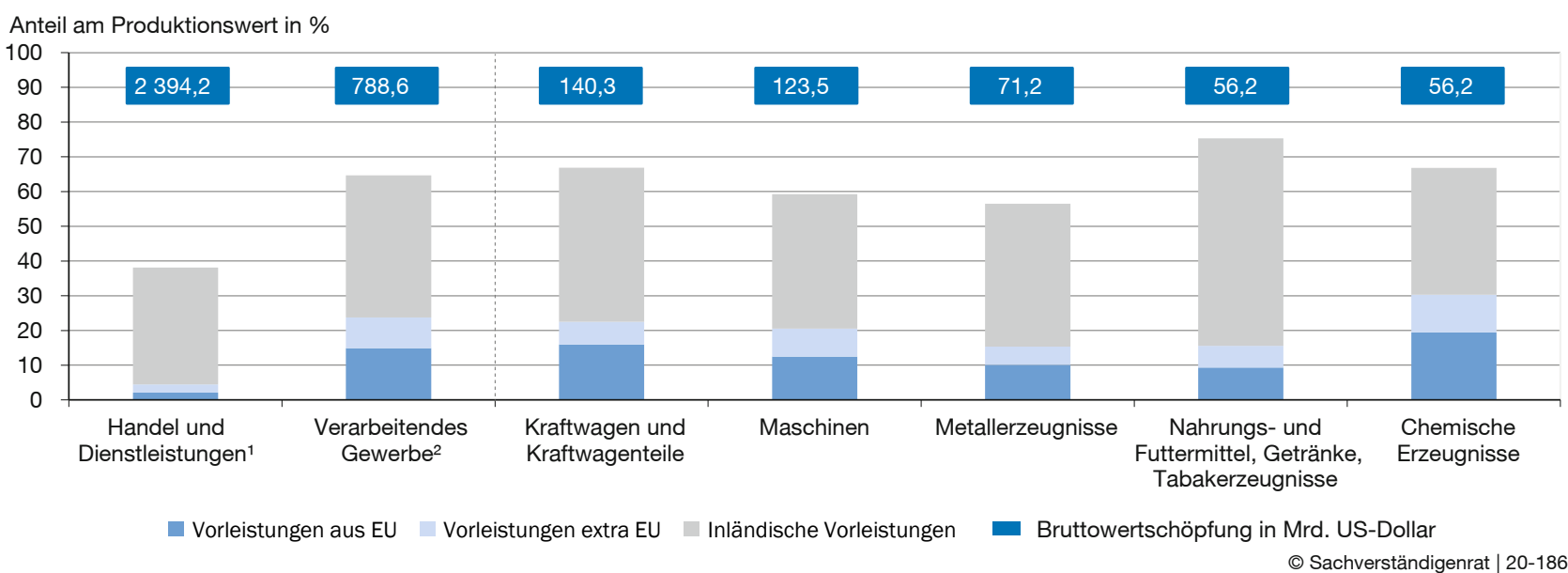

${ }^{1}$ ISIC Rev. 4 Sektoren G bis S. ${ }^{2}$ ISIC Rev. 4 Sektor C.

Quelle: World Input Output Database auf Basis des Jahres 2014; eigene Berechnungen.

entfallen dabei deutlich mehr als die Hälfte der Vorleistungsimporte auf EU-Mitgliedstaaten.

\section{Die große Bedeutung der Exportnachfrage für das Verarbeitende Gewerbe}

Nachfrageseitig gibt es ebenfalls große Unterschiede zwischen dem Verarbeitenden Gewerbe sowie den Bereichen Handel und Dienstleistungen (vgl. Abbildung 2). Letztere bedienen primär die inländische Nachfrage, der Anteil von Exporten am Produktionswert beträgt dort lediglich $8 \%$. Im Gegensatz dazu ist über die Hälfte der Nachfrage im Verarbeitenden Gewerbe auf Exporte zurückzuführen. Das Verarbeitende Gewerbe ist also nicht nur über den Vorleistungsbezug, sondern ebenfalls über den Absatz von Vorleistungsund Endgütern intensiv in internationale Wertschöpfungsketten eingebunden. Anders als beim Bezug von Vorleistungen hält sich absatzseitig das Handelsvolumen mit EU-Mitgliedstaaten und mit Staaten außerhalb der EU ungefähr die Waage. Insbesondere in den bruttowertschöpfungsstärksten Wirtschaftszweigen, dem Kraftwagenbau und dem Maschinenbau, ist das Handelsvolumen mit Staaten außerhalb der EU deutlich höher. Nur im Nahrungs- und Futtermittelbereich spielen Exporte in andere EU-Mitgliedstaaten eine dominante Rolle.

Besonders ins Auge sticht die Heterogenität zwischen den Wirtschaftszweigen hinsichtlich der Zusammensetzung der Exporte in Vorleistungs- und Endgüterexporte. Entsprechend ihrer jeweiligen Position in den Wertschöpfungsketten spielen in der Metallverarbeitung und in der chemischen Industrie Vorleistungsexporte eine wichtige Rolle (Fries et al.,
2020). In der Automobilbranche und im Maschinenbau sind dagegen Endgüterexporte wichtiger.

Im europäischen Vergleich wird deutlich, dass das Verarbeitende Gewerbe in Deutschland mit einem Exportanteil von $56 \%$ deutlich stärker als in den anderen großen EU-Mitgliedstaaten Frankreich und Italien mit Exportanteilen von $47 \%$ beziehungsweise $41 \%$ von der Exportnachfrage abhängig ist. Nichtsdestotrotz ist auch auf der Nachfrageseite insgesamt eine enge Verflechtung innerhalb der EU sichtbar. So beträgt in 19 von 27 Mitgliedstaaten der Anteil von Exporten in andere EU-Mitgliedstaaten im Verarbeitenden Gewerbe mehr als $25 \%$ des Produktionswerts.

Angesichts der starken Verflechtung über den Export von Vorleistungs- und Endgütern spielt die wirtschaftliche Entwicklung in Deutschlands wichtigsten Absatzmärkten für die konjunkturelle Lage der deutschen Industrie eine herausgehobene Rolle. Anhand der Einkaufsmanagerindizes ist eine Eintrübung von erheblicher Intensität zu beobachten (vgl. Abbildung 3). Während im Februar 2020 vor allem China betroffen war, zeigen sich ab März die Auswirkungen der Pandemie in Europa sowie im April eine weitere Verschlechterung der Lage und darüber hinaus deutliche Auswirkungen in den USA.

Dies hat sich im März 2020 bereits in den ausländischen Auftragseingängen und Auslandsumsätzen des Verarbeitenden Gewerbes niedergeschlagen (vgl. Abbildung 4). Die Umsätze sanken in allen Wirtschaftszweigen im Vergleich zum Februar. Zudem sanken in allen Wirtschaftszweigen, außer dem sonstigen Fahrzeugbau, die Auftragseingänge, wodurch sich 
Abbildung 2

Exportorientierung der deutschen Wirtschaft

Anteil am Produktionswert in \%

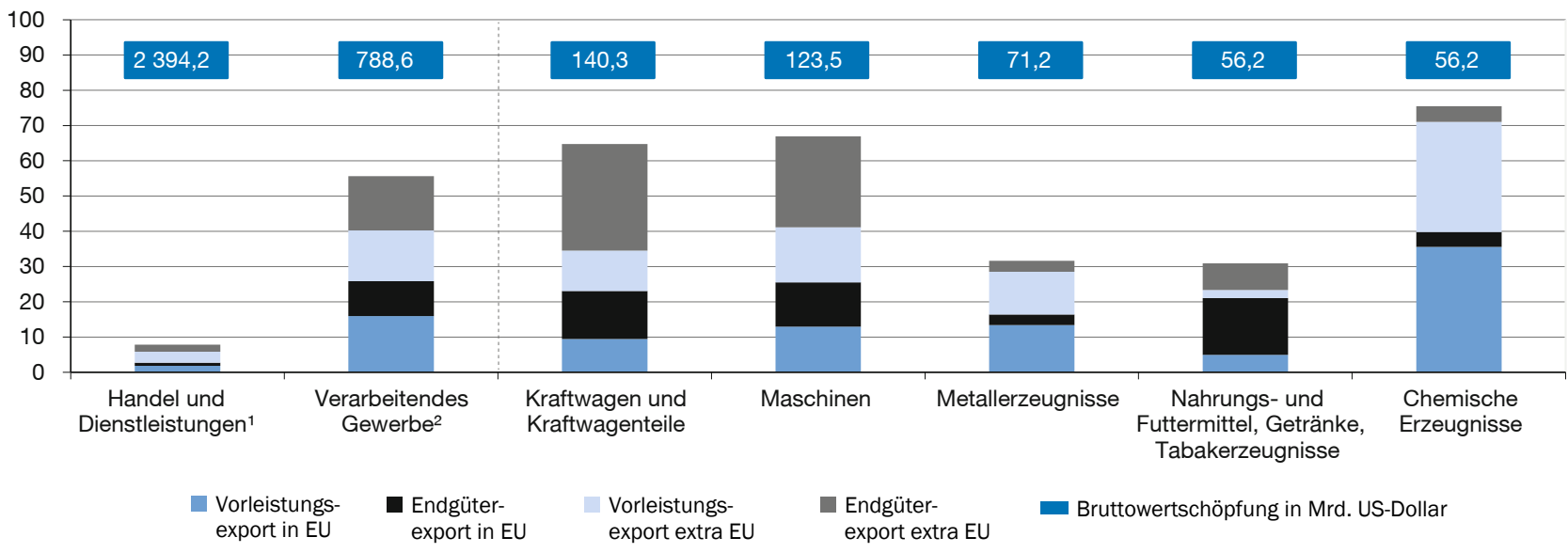

${ }^{1}$ ISIC Rev. 4, Sektoren G bis S. ${ }^{2}$ ISIC Rev. 4, Sektor C.

C Sachverständigenrat | 20-185

Quelle: World Input Output Database auf Basis des Jahres 2014; eigene Berechnungen.

die Zukunftsaussichten eintrübten. Am stärksten sind derzeit mit dem Maschinenbau und der Automobilindustrie zwei Branchen betroffen, die vornehmlich Güter der Endverwendung herstellen. In eher vorleistungsorientierten Branchen wie der chemischen Industrie oder der Metallverarbeitung sind bislang geringere Rückgänge zu beobachten. Insbesondere für die Wirtschaftszweige mit hohem Exportanteil stellen die Rückgänge der Auslandsnachfrage eine große Herausforderung dar. Hier dürfte eine Stimulierung der nationa- len Nachfrage nicht ausreichen, um den Nachfrageeinbruch aus dem Ausland zu kompensieren.

\section{Europäische Wirtschaftsmaßnahmen in der Corona-Krise}

Die starke Verflechtung der Lieferketten innerhalb der EU wirft die Frage nach der Rolle der EU in der Krisenbewältigung auf. Als schnelle und direkte Antwort auf die Ausbrei-

\section{Abbildung 3}

\section{Entwicklung des Verarbeitenden Gewerbes bei Deutschlands wichtigsten Handelspartnern ${ }^{1}$}

\section{Einkaufsmanagerindex (PMI), Indexpunkte}

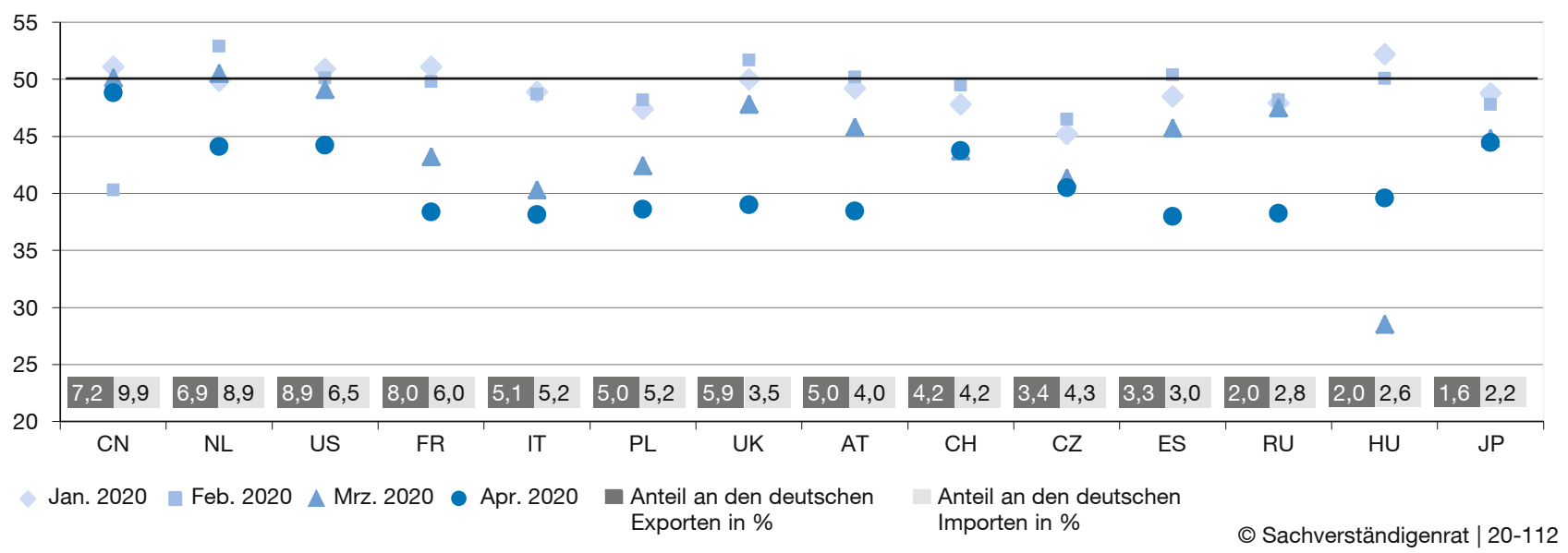

1 Dargestellt sind die 15 Partnerländer mit den höchsten Handelsumsätzen außer Belgien, für das keine Daten des Einkaufsmanagerindex verfügbar sind. Länderkürzel nach ISO-3166 Alpha-2: CN-Volksrepublik China, NL-Niederlande, US-USA, FR-Frankreich, IT-Italien, PL-Polen, UK-Vereinigtes Königreich, AT-Österreich, CH-Schweiz, CZ-Tschechische Republik, ES-Spanien, RU-Russische Föderation, HU-Ungarn, JP-Japan.

Quellen: Credit Suisse; Hungarian Association of Logistics, Purchasing and Inventory Management (HALPIM); Information Handling Services (IHS Markit); Statistisches Bundesamt. 


\section{Abbildung 4}

\section{Exporte und konjunktureller Einbruch von Februar bis März 2020 im Verarbeitenden Gewerbe}
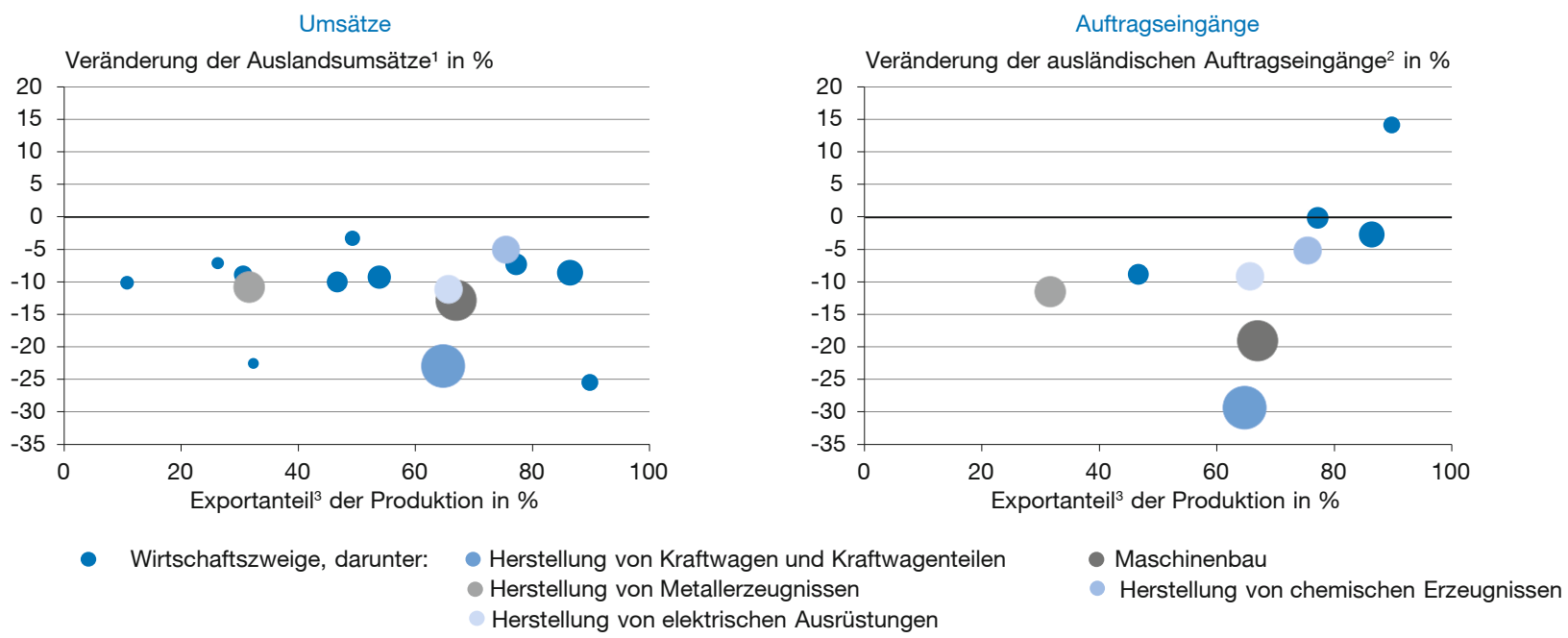

$\begin{array}{ll}\text { - Wirtschaftszweige, darunter: } & \text { Herstellung von Kraftwagen und Kraftwagenteilen } \\ & \text { Herstellung von Metallerzeugnissen } \\ & \text { Herstellung von elektrischen Ausrüstungen }\end{array}$

Kreisgröße proportional zur Bruttowertschöpfung der Wirtschaftszweige

(C) Sachverständigenrat | 20-174

${ }^{1}$ Veränderung der saison- und kalenderbereinigten Umsätze mit dem Ausland von Februar 2020 bis März 2020 in \%. ${ }^{2}$ Veränderung der saison- und kalenderbereinigten Auftragseingänge aus dem Ausland von Februar 2020 bis März 2020 in \%. ${ }^{3}$ Anteil der Exporte an der Gesamtproduktion des Wirtschaftszweigs im Jahr 2014 gemäß World Input Output Database in \%.

Quellen: Statistisches Bundesamt; World Input Output Database; eigene Berechnungen.

tung der Pandemie haben zunächst die Mitgliedstaaten Maßnahmen ergriffen, in deren Verantwortungsbereich auch die Gesundheitspolitik liegt. Die schnellere Reaktionszeit sowie die Möglichkeit, Maßnahmen bei einer heterogenen und zeitversetzten Ausbreitung zielgenau durchzuführen, spricht für ein Handeln auf nationaler oder sogar subnationaler Ebene.

Im Hinblick auf die Lieferketten und den Binnenmarkt kann aber Europa den größten Beitrag zur Überwindung der Krise und zum Wiederbeleben der gemeinsamen Wirtschaftsaktivität leisten. Insbesondere sind einheitliche und transparente sowie nachvollziehbare Vorgaben wichtig, um die Erwartungen der Unternehmen zu fokussieren und Planbarkeit bei der Wiederaufnahme der Produktion zu gewährleisten.

Die finanziellen Möglichkeiten der EU-Kommission sind jedoch begrenzt. Der aktuelle mehrjährige Finanzrahmen (MFR) endet in diesem Jahr. Die noch verbliebenen Mittel können von der Kommission, wie in ihrem ersten Maßnahmenpaket, lediglich umgewidmet oder vorgezogen werden. Finanzielle Ressourcen für die Zeit ab dem Jahr 2021 sind vom neuen MFR abhängig. Der aktuelle Plan für die Zeit bis 2027 umfasst 1.087 Mrd. Euro. Eine Einigung zwischen den Mitgliedstaaten auf den Umfang sowie die Verwendung konnte bislang nicht erreicht werden. Maßnahmen mit einem größeren Umfang wie das Kurzarbeiterprogramm SURE mit
100 Mrd. Euro oder der Garantiefonds der Europäischen Investitionsbank (EIB) mit 200 Mrd. Euro bedürfen einer gesonderten Mittelbereitstellung durch die Mitgliedstaaten. Die Kommission selbst kann hierbei nur koordinieren.

Größeren Spielraum für finanzielle Maßnahmen durch die EU-Kommission dürfte der von den Regierungschefs Frankreichs und Deutschlands vorgeschlagene Fonds eröffnen. Die in diesem Vorschlag enthaltene Kreditaufnahme durch die EU in Höhe von 500 Mrd. Euro soll der EUKommission die Möglichkeit geben, durch Zuschüsse anstatt ausschließlich durch Kredite die Folgen der CoronaPandemie zu addressieren. Da die Rückzahlung über den europäischen Haushalt erfolgen soll, liegt zumindest keine gesamtschuldnerische Haftung vor. Für die Umsetzbarkeit der Lösung dürfte es entscheidend sein, dass die Schuldenaufnahme tatsächlich einmalig und temporär geschehen soll, die Schulden also mit einem Tilgungsplan wieder zurückgezahlt werden.

Einen deutlich größeren Einfluss hat die EU-Kommission im Bereich nichtfinanzieller Maßnahmen. Die flexible Auslegung der Beihilferegelungen sowie der Haushaltsregeln ermöglicht den EU-Mitgliedstaaten eine deutlich stärkere Unterstützung der Wirtschaft. Erleichterungen für Finanzinstitute durch das Bankenpaket erlauben eine Ausweitung von Krediten und somit die Versorgung der Unternehmen mit Liquidität. In Bezug auf den Handel mit Staaten außer- 
halb der EU hat die Kommission etwa staatliche Exportkredite ermöglicht und Einfuhrzölle für medizinische Produkte ausgesetzt. Lockerungen in den Bereichen Beihilfe und Bankenregulierung sollten mittelfristig jedoch wieder zurückgenommen werden, um keine langfristigen Stabilitätsrisiken aufzubauen.

Für das Funktionieren der innereuropäischen Lieferketten hat die EU-Kommission eine wichtige aber eingeschränkte Rolle. Der Lieferverkehr innerhalb Europas ist durch die temporären Grenzschließungen beeinträchtigt. Die Kommission ist daher bemüht, die Einschränkungen so gering wie möglich zu halten. Dazu hat sie verschiedene Leitlinien an die Mitgliedstaaten ausgegeben, wie etwa den Grünen Korridor, der alle Gesundheits- und Einreisekontrollen bei Frachtfahrzeugen an den Grenzen in maximal 15 Minuten garantieren soll. Zudem soll keine Beschränkung des Warenverkehrs zwischen den Mitgliedsländern, wie zu Beginn der Krise für medizinische Güter, vorgenommen werden. Eine einheitliche Vorgehensweise der Staaten an der Grenze ist wichtig, um den bürokratischen Aufwand so gering wie möglich zu halten. Die EU-Kommission hat dazu Leitlinien für Grenzmanagementmaßnahmen veröffentlicht.

Zu Beginn der Corona-Pandemie haben die EU-Mitgliedstaaten mit erheblichen Einschränkungen der Personenreisefreiheit reagiert. Zwar wurde die Grenzüberschreitung aus beruflichen Gründen nicht untersagt, die Kontrollen haben jedoch zu erheblichen Belastungen für Berufstätige geführt. Grenzüberschreitungen zu Konsumzwecken waren weitestgehend verboten. Mittlerweile wurden einige dieser Beschränkungen wieder gelockert. Zur Sicherstellung der Reisefreiheit von Arbeitnehmern innerhalb des Schengen-Raums gelten grundsätzlich die Leitlinien für die Wahrung der Freizügigkeit systemrelevanter Arbeitskräfte. Weitere Leitlinien etwa für eine zügigere Anerkennung von Berufsqualifikationen des Gesundheitspersonals sollen in der aktuellen Gesundheitskrise für Entlastung sorgen. Die Beschränkung von nicht unbedingt notwendigen Reisen in die EU ist nach einer Aufforderung der EU-Kommission von allen beteiligten Ländern umgesetzt. Auch hierbei handelt es sich um Leitlinien, die Entscheidungen liegen jedoch bei den jeweiligen Mitgliedstaaten.

Die Leitlinien in den Bereichen des Güter- und Personenverkehrs sind für die Aufrechterhaltung der Lieferketten wichtig. Darüber hinaus könnte eine stärkere Koordination durch mehr Kompetenzen auf europäischer Ebene für weniger Unsicherheit und Bürokratie sorgen und einen noch reibungsloseren Ablauf an den Grenzen gewährleisten. Eine Ausdifferenzierung der Regeln in diesem Bereich ist lediglich bei regional unterschiedlichem Pandemieverlauf begründet. Während die jeweiligen Mitgliedstaaten möglicherweise zielgenauer und direkter Unternehmen finanziell und vor Ort unterstützen können, sollte der Grenzverkehr europaweit einheitlich geregelt werden.

\section{Wertschöpfungsketten europäisch wiederbeleben}

Die Bewältigung der Corona-Pandemie erfordert nach der ersten Phase der Eindämmung des Infektionsgeschehens ein gutes Zusammenspiel der verschiedenen Ebenen in Europa, um internationale Wertschöpfungsketten und somit auch die Wirtschaftsaktivität insgesamt weitestmöglich wiederzubeleben. Wo die internationale Verflechtung bedeutsam ist, müssen die Rahmenbedingungen auch auf europäischer Ebene einheitlich gesetzt werden. Dies betrifft insbesondere das Verarbeitende Gewerbe, das durch Vorleistungen und Exporte stark in internationale, insbesondere aber europäische Lieferketten eingebunden ist. Hier ist eine europäische Strategie unerlässlich. Können Probleme auf nationalstaatlicher oder regionaler Ebene effektiv adressiert werden, so ist dies vorzuziehen. Handelsund Dienstleistungsunternehmen sind nur in geringem Maße von der internationalen Lage abhängig und könnten ihre Aktivitäten wieder aufnehmen, sobald die Beschränkungen und die Konsumzurückhaltung wegfallen; die Wiederbelebung stellt dort somit eine vorwiegend nationale Herausforderung dar.

Um die Wirtschaftsaktivität im Verarbeitenden Gewerbe in allen EU-Staaten schnellstmöglich wiederzubeleben, ergibt sich dringender Handlungsbedarf auf EU-Ebene. An erster Stelle steht die gemeinsame Anstrengung zur Pandemiebekämpfung und dabei die Koordination und Abstimmung einheitlicher und transparenter gesundheitspolitischer Vorgaben. Zweitens sollten alle Staaten in die Lage versetzt werden, ihre Unternehmen durch die Krise zu bringen und die Wirtschaft wieder anzukurbeln. Zusätzliche Belastungen, Steuererhöhungen und Austerität in einzelnen Ländern könnten die konjunkturelle Wiederbelebung europaweit erschweren und somit die wirtschaftlichen Kosten der Corona-Pandemie signifikant erhöhen. Dies zu verhindern sollte angesichts der engen Verflechtung im gemeinsamen Interesse aller EU-Mitgliedstaaten sein.

\section{Literatur}

Bachmann, R. und C. Bayer (2013), 'Wait-and-See' business cycles?, Journal of Monetary Economics, 60(6), 704-719.

Flach, L., R. Aichele und M. Braml (2020), Status quo und Zukunft globaler Lieferketten, ifo Schnelldienst, 73(5), 16-22.

Fries, J. L. et al. (2020), Nachfrage- und angebotsseitige Einschränkungen der wirtschaftlichen Aktivität in Deutschland infolge der CoronaPandemie, Arbeitspapier, 2/2020, Sachverständigenrat zur Begutachtung der gesamtwirtschaftlichen Entwicklung, Wiesbaden.

Timmer, M. P., E. Dietzenbacher, B. Los, R. Stehrer und G. J. de Vries (2015), An Illustrated User Guide to the World Input-Output Database: the Case of Global Automotive Production, Review of International Economics, 23(3), 575-605. 